\title{
Shikonin inhibits inflammation and chondrocyte apoptosis by regulation of the PI3K/Akt signaling pathway in a rat model of osteoarthritis
}

\author{
DAIJIE FU, XIFU SHANG, ZHE NI and GUOGUANG SHI \\ Department of Orthopedics, Anhui Provincial Hospital, Hefei, Anhui 230001, P.R. China
}

Received April 30, 2015; Accepted July 5, 2016

DOI: $10.3892 /$ etm. 2016.3642

\begin{abstract}
Shikonin has previously been shown to have antitumor, anti-inflammatory, antiviral and extensive pharmacological effects. The aim of the present study was to explore whether the protective effect of shikonin is mediated via the inhibition of inflammation and chondrocyte apoptosis, and to elucidate the potential molecular mechanisms in a rat model of osteoarthritis. A model of osteoarthritis was established in healthy male Sprague-Dawley rats and $10 \mathrm{mg} / \mathrm{kg} / \mathrm{day}$ shikonin was administered intraperitoneally for 4 days. It was found that shikonin treatment significantly inhibited inflammatory reactions in the rats with osteoarthritis. Osteoarthritis was found to significantly increase interleukin (IL)-1 $\beta$, tumor necrosis factor (TNF)- $\alpha$ and inducible nitric oxide synthase (iNOS) levels compared with those in the sham group. However, shikonin treatment significantly inhibited the increases in IL-1 $\beta$, TNF- $\alpha$ and iNOS levels in the rats with osteoarthritis. Furthermore, caspase-3 activity and cyclooxygenase (COX)-2 protein expression were significantly increased and phosphorylated Akt protein expression was greatly suppressed in rats with osteoarthritis when compared with the sham group. Shikonin administration attenuated the changes in caspase-3 activity and COX-2 expression and Akt phosphorylation in rats with osteoarthritis. These results indicate that shikonin inhibits inflammation and chondrocyte apoptosis by regulating the phosphoinositide 3-kinase/Akt signaling pathway in a rat model of osteoarthritis.
\end{abstract}

\section{Introduction}

Osteoarthritis is one of the most common joint disorders affecting human health, with no evident racial and regional differences (1). Osteoarthritis is a cause of long-term disability

Correspondence to: Professor Xifu Shang, Department of Orthopedics, Anhui Provincial Hospital, 17 Lujiang Road, Hefei, Anhui 230001, P.R. China

E-mail: xifushang@126.com

Key words: shikonin, inflammation, chondrocyte apoptosis, phosphoinositide 3-kinase/Akt second only to cardiovascular disease in China (2). On the basis of a review, osteoarthritis giving rise to damage affects $2-6 \%$ of the population (3). Osteoarthritis has a greater influence on elderly patients than other diseases, affecting, for example, the ability to go up and down the stairs and other lower limb functions (4). Therefore, osteoarthritis is one of the major diseases leading to functional disability in people aged $>50$ years, causing loss to the economy and affecting social development (5).

Osteoarthritis is characterized by bone regeneration, and involves the degeneration and loss of articular cartilage with chronic arthritis of the joint edge and subchondral bone (6). The initial site of the disease is in the cartilage. The etiology and pathogenesis of osteoarthritis have not been fully elucidated; however, it is generally considered that systemic factors are involved, such as age, gender and family susceptibility, as well as local biomechanics, cartilage cell apoptosis, cell factors and the effects of degradative enzymes; it is a disease involving multiple linked factors (7). According to the literature, cartilage cell apoptosis may be important in the pathogenesis of osteoarthritis (8). At present, the signaling pathways involved in the induction of cartilage cell apoptosis, the regulation of signaling factors in such apoptosis, and indicators of chondrocyte apoptosis signal stimulation are topics of particular interest for experimental study. Numerous studies have confirmed that the pathogenic mechanism of osteoarthritis involves interleukin-1 (IL)-1, tumor necrosis factor (TNF)- $\alpha$ and matrix metalloproteinases (9). Through the determination of these indicators, the evolution process of osteoarthritis can be directly understood, particularly the association between osteoarthritis pathogenesis and cartilage cell apoptosis, which may be useful in the evaluation of targets and mechanisms for osteoarthritis intervention measures (10).

Lithospermum is the dry root of the borage perennial herbaceous plant Arnebia euchroma (Royle) Johnst. or Arnebia guttata Bunge (11). In China, it has a long medical history, and has been used clinically as a traditional Chinese medicine mainly for the treatment of wet macula, purpura, hematuria, dehydration, heat, constipation, burns, eczema and erysipelas, and for the promotion of circulation and removal of stasis (12). Shikonin is a naphthalene dione compound; studies have shown that shikonin plays an important role in the pharmacological effects of lithospermum, having anti-inflammatory and antibacterial effects, and inhibiting tumor proliferation 
and angiogenesis, thereby limiting tumor development (13). In the present study, whether shikonin exhibits a protective effect by inhibiting inflammation and chondrocyte apoptosis in a rat model of osteoarthritis was explored. In addition, the molecular mechanism of shikonin on osteoarthritis was investigated by researching changes in the phosphoinositide 3-kinase (PI3K)/Akt and mitochondrial signaling pathways.

\section{Materials and methods}

Materials. Shikonin was obtained from Sigma-Aldrich (St. Louis, MO, USA). IL-1 $\beta$, TNF- $\alpha$ and inducible nitric oxide synthase (iNOS) enzyme-linked immunosorbent assay (ELISA) kits were obtained from Beijing 4A Biotech Co., Ltd.(Beijing, China). This study was approved by the ethics committee of Anhui Provincial Hospital (Hefei, China).

Osteoarthritis animal model. Healthy male Sprague-Dawley rats $(\mathrm{n}=30 ; 8-10$-weeks old, 250-300 g), obtained from the Animal Science Laboratory of Anhui Provincial Hospital were anesthetized with $50 \mathrm{mg} / \mathrm{kg}$ pentobarbital intraperitoneally (i.p.) and shaved in a sterile state. Under sterile conditions, the right knee joint of the anesthetized rat was exposed through a medial parapatellar approach. Following anterior cruciate ligament transection and medial meniscus resection using micro-scissors, the patella was dislocated laterally and the knee was placed in full flexion. The rats were maintained under a 12 -h light/dark cycle at $22 \pm 2^{\circ} \mathrm{C}$ with $55 \pm 5 \%$ humidity, and were allowed free access to food and water.

Experimental groups and treatment. The rats were randomly assigned to three groups: Sham-operated group $(n=10)$, osteoarthritis model group $(\mathrm{n}=10)$ and shikonin-treated group $(n=10)$. In the sham-operated group, the right knee joint of the anesthetized rat was only exposed under sterile conditions, and the rats were treated with $0.1 \mathrm{ml} / 100 \mathrm{~g}$ physiological saline (i.p.). In the osteoarthritis model group, osteoarthritis model rats were treated with $0.1 \mathrm{ml} / 100 \mathrm{~g}$ physiological saline (i.p.). In the shikonin-treated group, osteoarthritis model rats were treated with $10 \mathrm{mg} / \mathrm{kg}$ shikonin (i.p.) once daily for 4 days after osteoarthritis modeling $(14,15)$.

ELISA analysis. Following treatment with $10 \mathrm{mg} / \mathrm{kg}$ shikonin, peripheral blood was collected from the abdominal aorta of rats in each group $(n=10)$. The blood was centrifuged at $12,000 \mathrm{x} \mathrm{g}$ for $10 \mathrm{~min}$ at $4^{\circ} \mathrm{C}$ and the supernatant was analyzed for IL- $1 \beta$, TNF- $\alpha$ and iNOS using ELISA assay kits according to the manufacturer's protocol (Beijing 4A Biotech Co., Ltd.).

Western blot analysis. Following the treatment with $10 \mathrm{mg} / \mathrm{kg}$ shikonin, rats were anesthetized with $50 \mathrm{mg} / \mathrm{kg}$ pentobarbital intraperitoneally (i.p.), sacrificed by decapitation, and samples of arthrotic tissue were collected ( $\mathrm{n}=10$ per group). The samples were homogenized with radioimmunoprecipitation assay (RIPA) lysis buffer (Beijing 4A Biotech Co., Ltd.). The homogenate was centrifuged at $12,000 \mathrm{x}$ g for $10 \mathrm{~min}$ at $4^{\circ} \mathrm{C}$ and analyzed using a bicinchoninic acid (BCA) assay kit (Beijing 4A Biotech Co., Ltd.). Approximately $50 \mu \mathrm{g}$ protein was separated by electrophoresis on a $12 \%$ sodium dodecyl sulfate (SDS)-polyacrylamide gel and then transferred onto a nitrocellulose filter membrane.

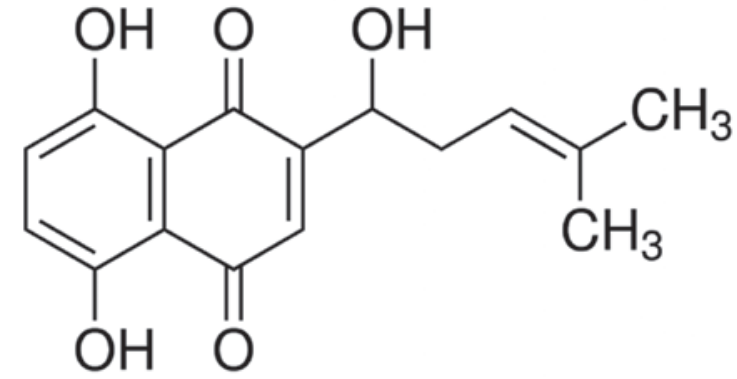

Figure 1. Chemical structure of shikonin.

Proteins were detected using mouse anti-nuclear factor (NF)- $\mathrm{\kappa B}$ p65 (sc-29311; 1:500), anti-cyclooxygenase (COX)-2 (sc-23984; 1:300), anti-Akt (sc-8312; 1:500) and anti-phosphorylated-Akt (anti-p-Akt; sc-135650; 1:1,000), all from Santa Cruz Biotechnology, Inc. (Dallas, TX, USA) and anti- $\beta$-actin (BB-2101-1; 1:5,000; BestBio Inc., Shanghai, China) followed by horseradish peroxidase-conjugated goat antimouse antibody (sc-2777; 1:5,000; Santa Cruz Biotechnology, Inc.). The relative quantities of protein expression were measured using AlphaEase FC (FluorChem FC2) software (ProteinSimple, Inc., San Jose, CA, USA).

Caspase-3 activity analysis. Following the 4-day treatment with $10 \mathrm{mg} / \mathrm{kg}$ shikonin, rats were sacrificed and osteoarthritis samples were collected. The samples were homogenized with RIPA lysis buffer. The homogenate was centrifuged at $12,000 \mathrm{x}$ g for $10 \mathrm{~min}$ at $4{ }^{\circ} \mathrm{C}$ and analyzed using a BCA assay kit. Protein $(20 \mu \mathrm{g})$ was mixed with the substrate Ac-DEVD-pNA (BB-4106-1; BestBio Inc.) in reaction buffer, and incubated at $37^{\circ} \mathrm{C}$ for $2 \mathrm{~h}$ in the dark. The absorption was then detected at a wavelength of $405 \mathrm{~nm}$.

Statistical analysis. All data are expressed as the mean \pm standard deviation. Data from each group were statistically analyzed by one-way analysis of variance followed by Tukey's tests. $\mathrm{P}<0.05$ was considered to indicate a statistically significant difference.

\section{Results}

Effect of shikonin on inflammation in the rat model of osteoarthritis. The chemical structure of shikonin is displayed in Fig. 1. To evaluate the anti-inflammatory effect of shikonin in a rat model of osteoarthritis, IL-1 $\beta$ and TNF- $\alpha$ levels were measured. As shown in Fig. 2, the expression levels of IL-1 $\beta$ and TNF- $\alpha$ were significantly increased in the rat model of osteoarthritis, compared with those in the sham group $(\mathrm{P}<0.01$; Fig. 2). However, shikonin significantly inhibited the increase in IL-1 $\beta$ and TNF- $\alpha$ expression levels in the rat model of osteoarthritis, compared with those in the osteoarthritis group (P<0.01; Fig. 2).

Effect of shikonin on NF- $\kappa B$ in the rat model of osteoarthritis. To appraise the anti-inflammatory mechanisms of shikonin in the rat model of osteoarthritis, the protein expression levels of $\mathrm{NF}-\kappa \mathrm{B}$ were examined using western blot analysis. There was a significant increase of the NF- $\kappa B$ protein expression level in 
A

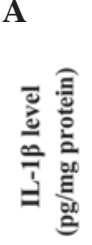

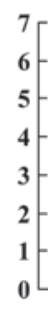

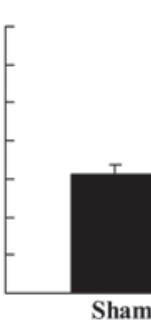

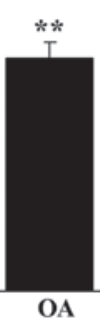

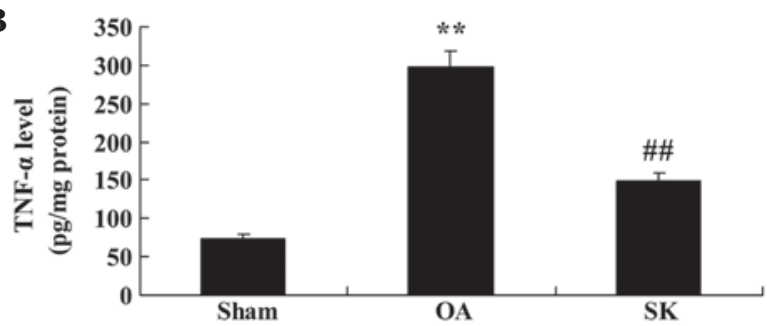

Figure 2. Effects of shikonin on inflammation in a rat model of osteoarthritis. (A) IL-1 $\beta$ and (B) TNF- $\alpha$ expression levels showing the effects of shikonin in a rat model of osteoarthritis. ${ }^{* *} \mathrm{P}<0.01$ vs. the sham group; ${ }^{\# \#} \mathrm{P}<0.01$ vs. the OA group. Sham, sham group; OA, osteoarthritis group; SK, shikonin group; IL-1 $\beta$, interleukin-1 $\beta$; TNF- $\alpha$, tumor necrosis factor- $\alpha$.

the rat model of osteoarthritis, compared with that in the sham group $(\mathrm{P}<0.01$; Fig. 3 ). However, the $\mathrm{NF}-\kappa \mathrm{B}$ protein expression level was significantly suppressed by shikonin in the rat model of osteoarthritis, compared with that in the osteoarthritis group $(\mathrm{P}<0.01$; Fig. 3$)$.

Effect of shikonin on iNOS level in the rat model of osteoarthritis. The effects of shikonin on the iNOS level in the rat model of osteoarthritis were investigated. The iNOS level in the rat model of osteoarthritis was significantly increased compared with that of the sham group $(\mathrm{P}<0.01$; Fig. 4$)$. The induction of the iNOS level was suppressed by treatment with shikonin in the rat model of osteoarthritis, compared with that in the osteoarthritis group $(\mathrm{P}<0.01$; Fig. 4).

Effect of shikonin on COX-2 expression in the rat model of osteoarthritis. To investigate the effects of shikonin on COX-2 in the rat model of osteoarthritis, COX-2 protein expression levels were measured by western blot analysis. The results showed that the protein expression level of COX-2 was markedly promoted in the rat model of osteoarthritis, as compared with that in the sham group $(\mathrm{P}<0.01$; Fig. 5). Notably, the administration of shikonin markedly weakened the upregulation of COX-2 protein expression in the rat model of osteoarthritis, as compared with that in the osteoarthritis group $(\mathrm{P}<0.01 ;$ Fig. 5$)$.

Effect of shikonin on caspase-3 activity in the rat model of osteoarthritis. In order to investigate the anti-apoptotic effect of shikonin on osteoarthritis, caspase-3 activity was measured. There was a significant increase in caspase- 3 activity in the rat model of osteoarthritis, as compared with the caspase-3 activity in the sham group $(\mathrm{P}<0.01$; Fig. 6). However, the elevation of caspase- 3 activity was significantly reduced by shikonin treatment in the rat model of osteoarthritis, compared with that in the osteoarthritis group $(\mathrm{P}<0.01$; Fig. 6).

Effect of shikonin on the phosphorylation of Akt in the rat model of osteoarthritis. The effects of shikonin on the phosphorylation of Akt in the rat model of osteoarthritis were examined; p-Akt expression levels were determined using western blot analysis. Notably, p-Akt protein expression of the osteoarthritic model group was lower than that of the sham group ( $\mathrm{P}<0.01$; Fig. 7). The downregulation of Akt phosphorylation was significantly recovered by treatment with shikonin in the rat model of osteoarthritis, compared with that in the osteoarthritis group $(\mathrm{P}<0.01$; Fig. 7$)$.
A

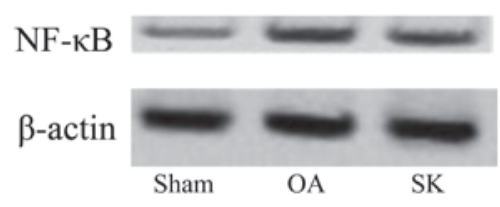

B

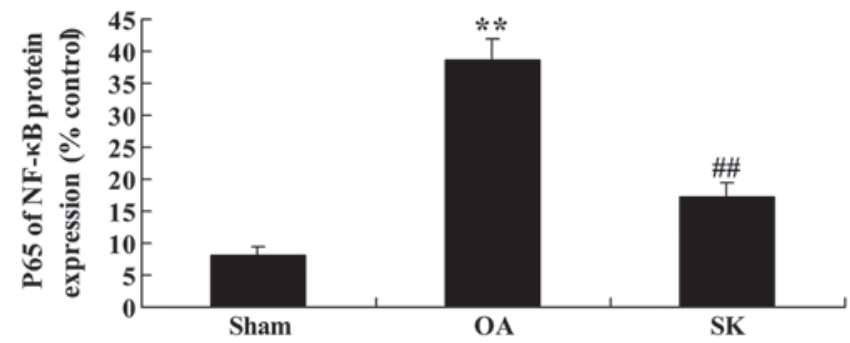

Figure 3. Effect of shikonin on NF- $\mathrm{BB}$ in a rat model of osteoarthritis. (A) Western blotting assay results and (B) statistical analysis of NF- $\mathrm{KB}$ protein expression levels showing the effect of shikonin on NF- $\kappa B$ protein in a rat model of osteoarthritis. ${ }^{* *} \mathrm{P}<0.01$ vs. the sham group; ${ }^{\# /} \mathrm{P}<0.01 \mathrm{vs}$. the OA group. Sham, sham group; OA, osteoarthritis group; SK, shikonin group; NF- $\kappa B$, nuclear factor- $\kappa \mathrm{B}$.

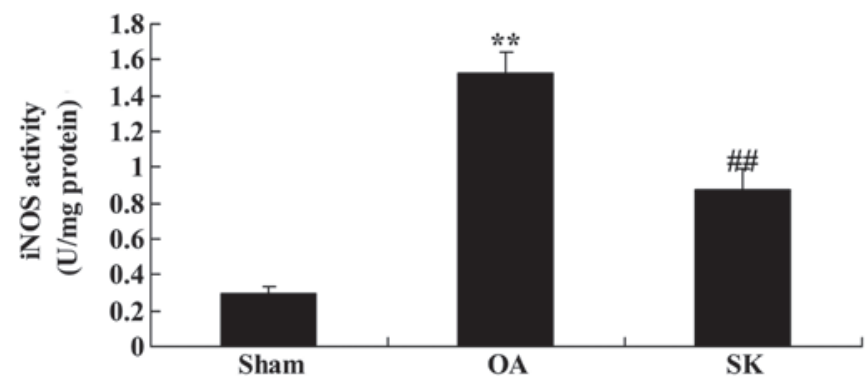

Figure 4. Effects of shikonin on iNOS activity in a rat model of osteoarthritis ${ }^{* *} \mathrm{P}<0.01$ vs. the sham group; ${ }^{\# \#} \mathrm{P}<0.01$ vs. the OA group. Sham, sham group; OA, osteoarthritis group; SK, shikonin group; iNOS, inducible nitric oxide synthase.

\section{Discussion}

Osteoarthritis is the most common chronic joint degenerative disease, and is also known as degenerative joint disease, hypertrophic arthritis and senile osteoarthropathy (16). The main pathological feature of osteoarthritis is degeneration of the articular cartilage, which results in articular cartilage injury, damage, joint edge and subchondral bone reactive hyperplasia and osteophyte formation (17). The incidence 
A

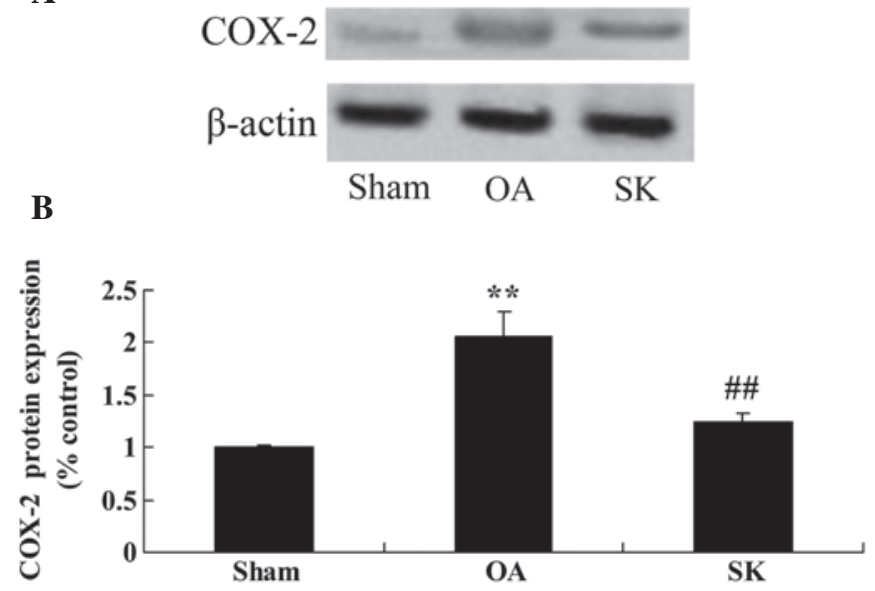

Figure 5. Effect of shikonin on COX-2 in a rat model of osteoarthritis. (A) Western blotting assay results and (B) statistical analysis of COX-2 protein expression levels showing the effect of shikonin in a rat model of osteoarthritis. ${ }^{* *} \mathrm{P}<0.01$ vs. the sham group; ${ }^{\# \#} \mathrm{P}<0.01$ vs. the OA group. Sham, sham group; OA, osteoarthritis group; SK, shikonin group; COX-2, cyclooxygenase 2 .

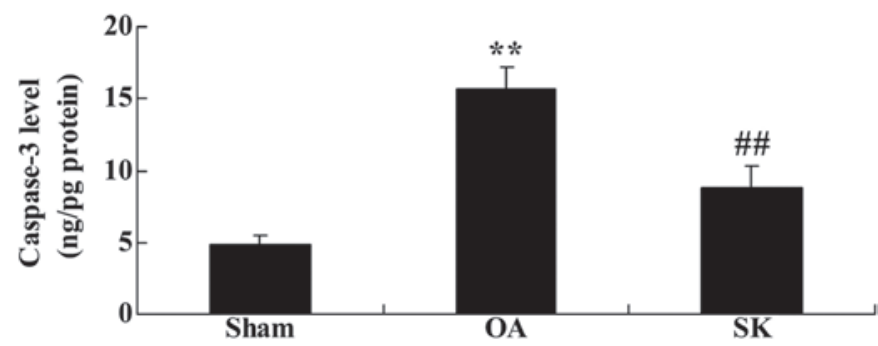

Figure 6. Effect of shikonin on caspase-3 activity in a rat model of osteoarthritis. ${ }^{* *} \mathrm{P}<0.01$ vs. the sham group; ${ }^{\# \#} \mathrm{P}<0.01$ vs. the OA group. Sham, sham group; OA, osteoarthritis group; SK, shikonin group.

of osteoarthritis is $3.0-8.3 \%$ in China, and in people aged $55-64$ years is $\sim 40 \%$ (18). However, the specific pathogenesis of osteoarthritis is unclear, and no reliable method to cure osteoarthritis clinically is available (19). The results of the present study revealed that shikonin treatment effectively inhibited the expression of IL- $1 \beta$ and TNF- $\alpha$ in a rat model of osteoarthritis. Furthermore, a previous study has indicated that shikonin exerts an anti-inflammatory effect via proteasome inhibition (14). Andújar et al (20) reported that shikonin exerts anti-inflammatory effects by inhibiting the activation of NF- $\mathrm{kB}$. These findings suggest that shikonin has an anti-inflammatory effect in rats with osteoarthritis.

$\mathrm{NF}-\mathrm{KB}$ is a nuclear transcription factor, widely present in eukaryotes, which plays a role in the central control of cell processes including inflammation, immune response, differentiation, proliferation, apoptosis and tumorigenesis (21). In the synthesis of various cytokines in the body, NF- $\kappa \mathrm{B}$ has a regulatory role in transcription. Under normal circumstances $\mathrm{NF}-\kappa \mathrm{B}$ and inhibitor of $\kappa \mathrm{B}$ (Iкb) combine and exist in the cytoplasm in an inactive form. When an activating signal is received, Ikb is phosphorylated, which releases NF-kB; at this point NF- $\mathrm{KB}$ is in an activated state (22). Following activation, $\mathrm{NF}-\kappa \mathrm{B}$ translocates to the nucleus and induces target gene
A

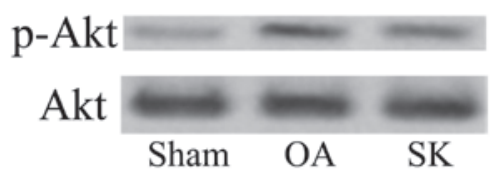

B

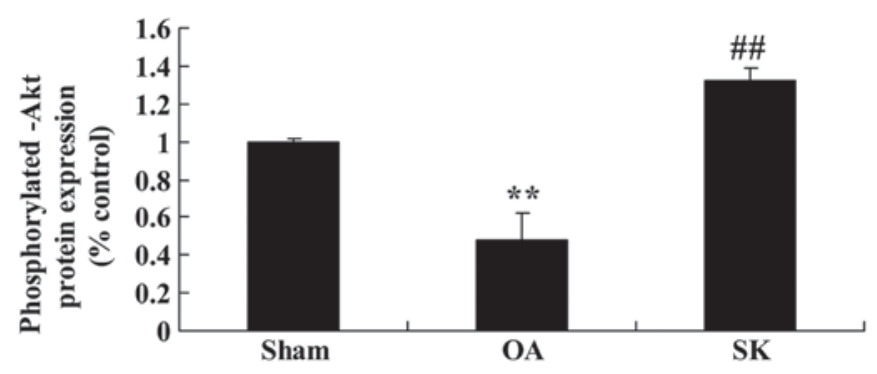

Figure 7. Effects of shikonin on p-Akt levels in a rat model of osteoarthritis. (A) Western blotting results and (B) statistical analysis of p-Akt protein expression levels showing the effect of shikonin in a rat model of osteoarthritis. ${ }^{* *} \mathrm{P}<0.01$ vs. the sham group; ${ }^{\# \#} \mathrm{P}<0.01$ vs. the OA group. Sham, sham group; OA, osteoarthritis group; $\mathrm{SK}$, shikonin group; p-Akt, phosphorylated Akt.

transcription, so as to regulate the synthesis of cytokines and other inflammatory factors (23). Studies have confirmed that NF- $\kappa \mathrm{B}$ has extensive biological effects, plays a significant role in the regulation of inflammation, and is a key link in the complex network of inflammatory cytokines $(24,25)$. Moreover, it modulates the occurrence and development of inflammation. For instance, NF- $\kappa \mathrm{B}$ is able to increase the transcription levels of TNF- $\alpha$ and IL-1, causing the secretion of these two cytokines to increase (26). Animal experiments have shown that in arthritis development, the activation of the NF- $\mathrm{KB}$ precedes the clinical manifestations of arthritis (27). In the present study, the anti-inflammatory action of shikonin suppressed NF- $\mathrm{\kappa B}$ protein expression in a rat model of osteoarthritis. Yang et al (28) reported that shikonin inhibited inflammation in RAW264.7 cells through NF- $\mathrm{BB}$ signaling pathways. Andújar et al (20) reported that shikonin exerted anti-inflammatory effects by inhibiting NF- $\mathrm{kB}$ activation. These results indicate that the anti-inflammatory activity of shikonin may be mediated via the downregulation of NF- $\mathrm{KB}$ signaling pathways in rats with osteoarthritis.

Apoptosis is a genetically regulated process of programmed cell death occurring in multicellular organisms; the regulatory mechanism of cell apoptosis-related gene expression is complex (29). The removal of cells by apoptosis is necessary for multicellular organisms to sustain life. There are numerous studies in which tumor cell proliferation is inhibited through apoptosis induction, or cell apoptosis is inhibited to repress chronic inflammation and potentially achieve anti-aging effects (30). In normal circumstances, the proliferation and apoptosis of articular cartilage cells are in dynamic equilibrium, which keeps the cell number, morphology and function of the articular cartilage generally stable; excessive apoptosis is pathological and harmful (31). Chondrocyte apoptosis has been confirmed to occur in articular cartilage affected by osteoarthritis (32). In addition, the excessive apoptosis of cartilage cells is considered to be one of the pathological factors in the 
degenerative changes of articular cartilage; thus cartilage cell apoptosis may be an important factor in the pathogenesis of osteoarthritis (33). Therefore, when considering how to reduce the excessive apoptosis of cartilage cells in order to prevent and treat osteoarthritis, maintaining a balance of cartilage cell proliferation and apoptosis is key (34). The present study showed that shikonin treatment significantly suppressed iNOS level elevation, COX-2 protein expression upregulation and increased caspase-3 activity in a rat model of osteoarthritis. Prasad et al (35) indicated that in lipopolysaccharide-stimulated BV2 microglial cells, shikonin downregulated the gene expression of proinflammatory NOS and COX-2. Yang et al (28) reported that shikonin protects against interleukin-1 $\beta$-induced apoptosis in chondrocytes via the inhibition of caspase-3 activity. Thus, the aforementioned results suggest that iNOS, COX-2 and caspase- 3 are important molecular targets of shikonin in the treatment of osteoarthritis.

The mechanism of cartilage cell apoptosis is complex and has not been fully elucidated. However, it may be associated with various signal transduction pathways, among which the $\mathrm{PI} 3 \mathrm{~K} /$ Akt signal transduction pathway is regarded as an important pathway of cartilage cell apoptosis (36). As a membrane protein, $\mathrm{PI} 3 \mathrm{~K}$ receives incoming signals from tyrosine kinase receptors, cytokine receptors, CD19, B-cell receptors and G-protein-coupled receptors, which directly or indirectly activates Akt and downstream factors (37). Akt has functions in protein synthesis, cell apoptosis, cell cycle regulation, glucose metabolism and nerve degeneration (38). The current study demonstrates that treatment with shikonin significantly activated the downregulation of Akt activation in a rat model of osteoarthritis. Huang et al (39) found that shikonin inhibited oxidized low-density lipoprotein-induced monocyte adhesion through the upregulation of PI3K/Akt and the suppression of $\mathrm{NF}-\kappa \mathrm{B}$ activation. Kamei et al (40) suggested that in 3T3-L1 adipocytes, shikonin stimulated glucose uptake via a mechanism involving Akt phosphorylation. The results of the present study indicate that shikonin has anti-inflammatory effects in rats with osteoarthritis and one of the underlying mechanisms may be the upregulation of PI3K/Akt signaling pathways.

In conclusion, the results of the present study confirmed that shikonin inhibits inflammation and chondrocyte apoptosis by regulating the PI3K/Akt signaling pathway in a rat model of osteoarthritis. These findings suggest that shikonin has therapeutic potential for osteoarthritis.

\section{References}

1. Wu C, Tian B, Qu X, Liu F, Tang T, Qin A, Zhu Z and Dai K MicroRNAs play a role in chondrogenesis and osteoarthritis (review). Int J Mol Med 34: 13-23, 2014.

2. Yu H, Wang Y, Guo Y, Wang H, Chen B and Zhao X: Quality assessment of randomized controlled trials reporting on knee osteoarthritis treated with warming needle moxibustion. J Tradit Chin Med 34: 621-626, 2014.

3. Wang X, Wei S, Liu T, Pang J, Gao N, Ding D, Duan T, Cao Y, Zheng Y and Zhan H: Effectiveness, medication patterns and adverse events of traditional Chinese herbal patches for osteoarthritis: A systematic review. Evid Based Complement Alternat Med 2014: 343176, 2014.

4. Baker KR, Xu L, Zhang Y, Nevitt M, Niu J, Aliabadi P, Yu W and Felson D: Quadriceps weakness and its relationship to tibiofemoral and patellofemoral knee osteoarthritis in Chinese: The Beijing osteoarthritis study. Arthritis Rheum 50: 1815-1821, 2004.
5. Robertson C, Archibald D, Avenell A, Douglas F, Hoddinott P, van Teijlingen E, Boyers D, Stewart F, Boachie C, Fioratou E, et al: Systematic reviews of and integrated report on the quantitative, qualitative and economic evidence base for the management of obesity in men. Health Technol Assess 18: v-vi, xxiii-xxix, 1-424, 2014.

6. Lu H, Hou G, Zhang Y, Dai Y and Zhao H: C-Jun transactivates Puma gene expression to promote osteoarthritis. Mol Med Rep 9: 1606-1612, 2014.

7. Patel DV, Sawant MG and Kaur G: Evaluation of anti-osteoarthritic activity of Vigna mungo in papain induced osteoarthritis model. Indian J Pharmacol 47: 59-64, 2015.

8. Wang F, Wu L, Li L and Chen S: Monotropein exerts protective effects against IL-1 $\beta$-induced apoptosis and catabolic responses on osteoarthritis chondrocytes. Int Immunopharmacol 23: 575-580, 2014.

9. Zangerle PF, De Groote D, Lopez M, Meuleman RJ, Vrindts Y, Fauchet F, Dehart I, Jadoul M, Radoux D and Franchimont P: Direct stimulation of cytokines (IL-1 beta, TNF-alpha, IL-6, IL-2, IFN-gamma and GM-CSF) in whole blood: II. Application to rheumatoid arthritis and osteoarthritis. Cytokine 4: 568-575, 1992.

10. Zamli Z, Robson Brown K, Tarlton JF, Adams MA, Torlot GE, Cartwright C, Cook WA, Vassilevskaja K and Sharif M: Subchondral bone plate thickening precedes chondrocyte apoptosis and cartilage degradation in spontaneous animal models of osteoarthritis. Biomed Res Int 2014: 606870, 2014.

11. Wang $X$, Hayashi S, Umezaki M, Yamamoto $T$, Kageyama-Yahara N, Kondo T and Kadowaki M: Shikonin, a constituent of Lithospermum erythrorhizon exhibits anti-allergic effects by suppressing orphan nuclear receptor $\mathrm{Nr} 4 \mathrm{a}$ family gene expression as a new prototype of calcineurin inhibitors in mast cells. Chem Biol Interact 224C: 117-127, 2014.

12. Wada N, Kawano Y, Fujiwara S, Kikukawa Y, Okuno Y, Tasaki M, Ueda M, Ando Y, Yoshinaga K, Ri M, et al: Shikonin, dually functions as a proteasome inhibitor and a necroptosis inducer in multiple myeloma cells. Int J Oncol 46: 963-972, 2015.

13. Wang R, Yin R, Zhou W, Xu D and Li S: Shikonin and its derivatives: A patent review. Expert Opin Ther Pat 22: 977-997, 2012.

14. Lu L, Qin A, Huang H, Zhou P, Zhang C, Liu N, Li S, Wen G, Zhang C, Dong W, et al: Shikonin extracted from medicinal Chinese herbs exerts anti-inflammatory effect via proteasome inhibition. Eur J Pharmacol 658: 242-247, 2011.

15. Öberg AI, Yassin K, Csikasz RI, Dehvari N, Shabalina IG, Hutchinson DS, Wilcke M, Östenson CG and Bengtsson T: Shikonin increases glucose uptake in skeletal muscle cells and improves plasma glucose levels in diabetic Goto-Kakizaki rats. PLoS One 6: e22510, 2011.

16. Fox BA and Stephens MM: Glucosamine/chondroitin/primorine combination therapy for osteoarthritis. Drugs Today (Barc) 45: 21-31, 2009.

17. Cicero AF and Laghi L: Activity and potential role of licofelone in the management of osteoarthritis. Clin Interv Aging 2: 73-79, 2007.

18. Guo D, Cao XW, Liu JW, Niu W, Ma ZW, Lin DK, Chen JY, Lian WD, Ouyang WW and Liu J: Clinical effectiveness and micro-perfusion alteration of Jingui external lotion in patients with knee osteoarthritis: Study protocol for a randomized controlled trial. Trials 16: 124, 2015.

19. Li Y, Zhang H, Zhang J, Li X, Song G and Feng H: Clinical outcome of simultaneous high tibial osteotomy and anterior cruciate ligament reconstruction for medial compartment osteoarthritis in young patients with anterior cruciate ligament-deficient knees: A systematic review. Arthroscopy 31: 507-519, 2015.

20. Andújar I, Recio MC, Bacelli T, Giner RM and Ríos JL: Shikonin reduces oedema induced by phorbol ester by interfering with IkappaBalpha degradation thus inhibiting translocation of NF-kappaB to the nucleus. Br J Pharmacol 160: 376-388, 2010.

21. Hilgendorff A, Muth H, Parviz B, Staubitz A, Haberbosch W, Tillmanns $\mathrm{H}$ and Hölschermann $\mathrm{H}$ : Statins differ in their ability to block NF-kappaB activation in human blood monocytes. Int J Clin Pharmacol Ther 41: 397-401, 2003.

22. Kwak SC, Lee C, Kim JY, Oh HM, So HS, Lee MS, Rho MC and Oh J: Chlorogenic acid inhibits osteoclast differentiation and bone resorption by down-regulation of receptor activator of nuclear factor kappa-B ligand-induced nuclear factor of activated T cells c1 expression. Biol Pharm Bull 36: 1779-1786, 2013.

23. Bowles RD, Mata BA, Bell RD, Mwangi TK, Huebner JL, Kraus VB and Setton LA: In vivo luminescence imaging of NF- $\kappa B$ activity and serum cytokine levels predict pain sensitivities in a rodent model of osteoarthritis. Arthritis Rheumatol 66: 637-646, 2014. 
24. Cho HJ, Lee KW and Park JH: Erucin exerts anti-inflammatory properties in murine macrophages and mouse skin: Possible mediation through the inhibition of $\mathrm{NF} \kappa \mathrm{B}$ signaling. Int $\mathrm{J}$ Mol Sci 14: 20564-20577, 2013.

25. Toegel S, Weinmann D, André S, Walzer SM, Bilban M, Schmidt S, Chiari C, Windhager R, Krall C, Bennani-Baiti IM and Gabius HJ: Galectin-1 couples glycobiology to inflammation in osteoarthritis through the activation of an NF- $\kappa$ B-regulated gene network. J Immunol 196: 1910-1921, 2016.

26. Ni S, Miao K, Zhou X, Xu NW, Li CK, Zhu RX, Sun RB and Wang YJ: The involvement of follistatin-like protein 1 in osteoarthritis by elevating $\mathrm{NF}-\kappa \mathrm{B}$-mediated inflammatory cytokines and enhancing fibroblast like synoviocyte proliferation. Arthritis Res Ther 17: 91, 2015.

27. Chan DD, Xiao WF, Li J, de la Motte CA, Sandy JD and Plaas A: Deficiency of hyaluronan synthase 1 (Has1) results in chronic joint inflammation and widespread intra-articular fibrosis in a murine model of knee joint cartilage damage. Osteoarthritis Cartilage 23: 1879-1889, 2015.

28. Yang Y, Wang J, Yang Q, Wu S, Yang Z, Zhu H, Zheng M, Liu W, Wu W, He J and Chen Z: Shikonin inhibits the lipopolysaccharide-induced release of HMGB1 in RAW264.7 cells via IFN and NF- $\kappa$ B signaling pathways. Int Immunopharmacol 19: 81-87, 2014.

29. Fang HY, Chen CY, Hung MF, Hsiao YT, Chiang TC, Lin TY, Chang HW, Chow KC and Ko WJ: Caspase-14 is an anti-apoptotic protein targeting apoptosis-inducing factor in lung adenocarcinomas. Oncol Rep 26: 359-369, 2011

30. Chan DD, Xiao WF, Li J, de la Motte CA, Sandy JD and Plaas A: Deficiency of hyaluronan synthase 1 (Hasl) results in chronic joint inflammation and widespread intra-articular fibrosis in a murine model of knee joint cartilage damage. Osteoarthritis Cartilage 23: 1879-1889, 2015.

31. Machner A, Baier A, Wille A, Drynda S, Pap G, Drynda A, Mawrin C, Bühling F, Gay S, Neumann W and Pap T: Higher susceptibility to Fas ligand induced apoptosis and altered modulation of cell death by tumor necrosis factor-alpha in periarticular tenocytes from patients with knee joint osteoarthritis. Arthritis Res Ther 5: R253-R261, 2003
32. Sezgin M, Barlas İÖ, Yıldır S, Türköz G, Ankaralı HÇ, Sahin G and Erdal ME: Apoptosis-related Fas and FasL gene polymorphisms' associations with knee osteoarthritis. Rheumatol Int 33: 2039-2043, 2013.

33. López-Armada MJ, Caramés B, Cillero-Pastor B, Lires-Deán M, Maneiro E, Fuentes I, Ruíz C, Galdo F and Blanco FJ: Phosphatase-1 and $-2 \mathrm{~A}$ inhibition modulates apoptosis in human osteoarthritis chondrocytes independently of nitric oxide production. Ann Rheum Dis 64: 1079-1082, 2005.

34. Takács-Buia L, Iordachel C, Efimov N, Caloianu M, Montreuil J and Bratosin D: Pathogenesis of osteoarthritis: Chondrocyte replicative senescence or apoptosis? Cytometry B Clin Cytom 74: 356-362, 2008

35. Prasad RG, Choi YH and Kim GY: Shikonin isolated from lithospermum erythrorhizon downregulates proinflammatory mediators in lipopolysaccharide-stimulated BV2 microglial cells by suppressing crosstalk between reactive oxygen species and NF- $\kappa$ B. Biomol Ther (Seoul) 23: 110-118, 2015.

36. $\mathrm{Yu} \mathrm{SM}$ and Kim SJ: Withaferin A-caused production of intracellular reactive oxygen species modulates apoptosis via PI3K/Akt and JNKinase in rabbit articular chondrocytes. J Korean Med Sci 29: 1042-1053, 2014.

37. Sugimori K, Matsui K, Motomura H, Tokoro T, Wang J, Higa S, Kimura T and Kitajima I: BMP-2 prevents apoptosis of the N1511 chondrocytic cell line through PI3K/Akt-mediated NF-kappaB activation. J Bone Miner Metab 23: 411-419, 2005.

38. Lee KN, Seo MC, Bae IH, Oh SH, Jang WG, Jeong BC, Oh WM, Kim SH, Lee SE and Shim KM, et al: COMP-Ang1, a variant of angiopoietin 1, inhibits serum-deprived apoptosis of mesenchymal cells via PI3K/Akt and mitogen-activated protein kinase pathways. Pharmacology 86: 327-335, 2010.

39. Huang CS, Lin AH, Yang TC, Liu KL, Chen HW and Lii CK: Shikonin inhibits oxidized LDL-induced monocyte adhesion by suppressing NFKB activation via up-regulation of PI3K/Akt/ Nrf2-dependent antioxidation in EA. hy926 endothelial cells. Biochem Pharmacol 93: 352-361, 2015.

40. Kamei R, Kitagawa Y, Kadokura M, Hattori F, Hazeki O, Ebina Y, Nishihara T and Oikawa S: Shikonin stimulates glucose uptake in 3T3-L1 adipocytes via an insulin-independent tyrosine kinase pathway. Biochem Biophys Res Commun 292: 642-651, 2002 